\title{
A COUNTEREXAMPLE TO THE TWO-THIRDS CONJECTURE
}

\author{
ROGER W. BARNARD AND JOHN L. LEWIS
}

\begin{abstract}
Let $w=f(z)=z+a_{2} z^{2}+\cdots$ be regular and univalent for $|z|<1$, and map $|z|<1$ onto a region which is starlike with respect to $w=0$. If $r_{0}$ denotes the radius of convexity of $w=$ $f(z), d^{*}=\min |f(z)|$ for $|z|=r_{0}$ and $d=\inf |\beta|$ for which $f(z) \neq \beta$, then it has been conjectured by A. Schild in 1953 that $d^{*} / d \geqq \frac{2}{3}$. It is shown here that this conjecture is false by giving two counterexamples.
\end{abstract}

1. Introduction. Let $S^{*}$ be the class of univalent starlike functions $f$ in $K=\{z:|z|<1\}$ with $f(0)=0$. Let $r_{0}=r_{0}(f)$ be the radius of convexity of $f$ (see Hayman [2] for a definition). Put $d^{*}=\min _{|z|=r_{0}}|f(z)|$ and $d=$ inf $|\beta|$ for which $f(z) \neq \beta$. Then in 1953, A. Schild [5] conjectured that $d^{*} / d \geqq \frac{2}{3}$. Here equality holds for $f(z)=z(1+z)^{-2}, z \in K$. Schild noted that $d^{*} / d \geqq r_{0} \geqq 2-\sqrt{ } 3$ (see Hayman [2, p. 141]) and proved the conjecture for $p$ symmetric functions, $p \geqq 7$. He also showed for a certain class of circularly symmetric functions that $d^{*} / d \geqq 0.49$. Lewandowski and others ([3], [1]) proved the conjecture true for certain subclasses of $S^{*}$. Recently McCarty and Tepper [4] have shown that $d^{*} / d \geqq 0.380$ for a function in $S^{*}$.

In this paper we disprove the two-thirds conjecture by giving two counterexamples. The first counterexample is given simply by

$$
f_{\alpha}(z)=z(1-z)^{-\alpha}(1+z)^{\alpha-2}, \quad z \in K, 0<\alpha<2,
$$

where $\alpha$ is sufficiently near zero. As motivation for this example, we note that if $d$ is computed as a function of $\alpha$, then $(d / d \alpha)(d) \rightarrow+\infty$ as $\alpha \rightarrow 0$ [see (2.2)]. For $\alpha=0.03$ we obtain $d^{*} / d \leqq 0.656$.

We also give an example of a circularly symmetric function in $S^{*}$ for which $d^{*} / d<0.645$. Therefore the two-thirds conjecture is false even for circularly symmetric functions.

Presented to the Society, January 26, 1973; received by the editors December 6 , 1972 and, in revised form, January 29, 1973.

AMS (MOS) subject classifications (1970). Primary 30A32.

Key words and phrases. Univalent functions, starlike functions, convex functions, radius of convexity. 
2. Example 1. For $f_{\alpha}(z)$ defined by (1.1), $w=f_{\alpha}(z)$ maps $K$ onto the entire $w$-plane minus two radial slits. The slits are symmetric about the positive real axis and are separated by an angle of $\alpha \pi$. From the mapping properties of $f_{\alpha}(z)$ it is clear that $d=\left|f_{\alpha}\left(z_{0}\right)\right|$ where $f_{\alpha}^{\prime}\left(z_{0}\right)=0$. Since

$$
p(z)=z f_{\alpha}^{\prime}(z) / f_{\alpha}(z)=\left[1+2(\alpha-1) z+z^{2}\right]\left(1-z^{2}\right)^{-1}, \quad z \in K,
$$

then $f_{\alpha}^{\prime}\left(z_{0}\right)=0$ if $z_{0}$ satisfies the equation $1+2(\alpha-1) z+z^{2}=0$. Hence $z_{0}=(1-\alpha) \pm i\left[1-(1-\alpha)^{2}\right]^{1 / 2}$. From symmetry we may choose either sign. Thus

$$
\log d(\alpha)=-\frac{1}{2} \alpha \log 2 \alpha+\frac{1}{2}(\alpha-2) \log (4-2 \alpha) .
$$

For fixed $\alpha, 0<\alpha \leqq 1$, let $r_{1}=r_{1}(\alpha)$ be the smallest positive root of the equation

$$
1+r f_{\alpha}^{\prime \prime}(r) / f_{\alpha}^{\prime}(r)=0, \quad 0<r<1 .
$$

Let $d_{1}=f_{\alpha}\left(r_{1}\right)$. Then $r_{1} \geqq r_{0}$ and consequently

$$
d^{*} \leqq d_{1},
$$

since the minimum modulus of $f_{\alpha}$ is increasing as a function of $r, 0<r<1$.

To obtain $r_{1}$ we solve (2.3). From (2.1) we have

$$
\begin{aligned}
1+\frac{z f_{\alpha}^{\prime \prime}(z)}{f_{\alpha}^{\prime}(z)} & =p(z)+\frac{z p^{\prime}(z)}{p(z)} \\
& =\frac{1+(6 \alpha-6) z+\left(4 \alpha^{2}-8 \alpha+10\right) z^{2}+(6 \alpha-6) z^{3}+z^{4}}{\left(1-z^{2}\right)\left[1+2(\alpha-1) z+z^{2}\right]} .
\end{aligned}
$$

Thus $r_{1}$ is the first positive root of

$$
\begin{aligned}
F(r, \alpha) & =1+6(\alpha-1) r+2\left(2 \alpha^{2}-4 \alpha+5\right) r^{2}+6(\alpha-1) r^{3}+r^{4} \\
& =0 .
\end{aligned}
$$

Substituting $u=r+r^{-1}$ in (2.5) we obtain a quadratic equation in $u$. The quadratic formula gives

$$
u_{1}=3(1-\alpha)+\left[5(1-\alpha)^{2}-4\right]^{1 / 2}, \quad r_{1}=\frac{1}{2}\left[u_{1}-\left(u_{1}^{2}-4\right)^{1 / 2}\right] .
$$

We note that

$$
\lim _{\alpha \rightarrow 0} r_{1}(\alpha)=2-\sqrt{3} \text { and } \lim _{\alpha \rightarrow 0} f_{\alpha}(z)=\frac{z}{(1+z)^{2}}
$$

uniformly on compact subsets of $K$. 
Using (2.2) and (*) it follows that $\lim _{\alpha \rightarrow 0} d_{1} / d=\frac{2}{3}$. Also $(d / d \alpha)\left(d_{1} / d\right)$ is a continuous function of $\alpha$ for $0<\alpha<1$, as is easily seen. From the mean value theorem of differential calculus, we conclude that if

$$
\lim _{\alpha \rightarrow 0} \frac{d}{d \alpha}\left(\frac{d_{1}}{d}\right)<0
$$

then the two-thirds conjecture is false. Now

$$
\begin{aligned}
\frac{d}{d \alpha} \log \frac{d_{1}(\alpha)}{d(\alpha)} & =\frac{d}{d \alpha} \log f_{\alpha}\left(r_{1}\right)-\frac{d}{d \alpha} \log d(\alpha) \\
& =\frac{d r_{1}}{d \alpha} \frac{f_{\alpha}^{\prime}\left(r_{1}\right)}{f_{\alpha}\left(r_{1}\right)}+\log \frac{1+r_{1}}{1-r_{1}}+\frac{1}{2} \log \frac{\alpha}{2-\alpha},
\end{aligned}
$$

thanks to (2.2). Since $\log [\alpha /(2-\alpha)] \rightarrow-\infty$ as $\alpha \rightarrow 0$ and $\left(^{*}\right)$ is true, it follows that we need only show $\lim _{\alpha \rightarrow 0}\left(d r_{1}(\alpha) / d \alpha\right)<+\infty$ to prove (2.7). This can be shown directly from (2.6) or by the following argument. The function $F(r, \alpha)$ defined in (2.5) has continuous first partials in $\alpha$ and $r$. Moreover $F(2-\sqrt{ } 3,0)=0$. From the implicit function theorem it follows that if $(\partial F / \partial r)(2-\sqrt{3}, 0) \neq 0$, then $d r / d \alpha$ is continuous in a neighborhood of zero. Since $(\partial F / \partial r)(2-\sqrt{ } 3,0)=12-8 \sqrt{ } 3 \neq 0$, we conclude that $(2.7)$ is true and thereupon, for $\alpha$ near $0, \alpha>0$, that $d^{*} \mid d \leqq d_{1} / d<\frac{2}{3}$.

A close approximation to the minimum of $d_{1}(\alpha) / d(\alpha)$ is given by $\alpha=0.03$. For $\alpha=0.03$ we obtain using (1.1), (2.2) and (2.6) that $d^{*} / d \leqq$ 0.656 .

3. Example 2. In this section we give an example of a circularly symmetric function for which $d^{*} / d<0.645$. We use the functions $g_{a}$, $-1<a<1$, which have been shown by $T$. Suffridge in [6] to solve an important extremal problem. Let $g_{a}$ be defined by

$$
\begin{aligned}
F(z) & =\left(z g_{a}^{\prime}(z)\right) /\left(g_{a}(z)\right) \\
& =\left[\left(1+2 a z+z^{2}\right) /(1-z)^{2}\right]^{1 / 2}, \quad z \in K,-1<a<1 .
\end{aligned}
$$

Since $(\partial / \partial \theta) \log g_{a}\left(e^{i \theta}\right)=i F\left(e^{i \theta}\right)$ (any branch of $\log g_{a}\left(e^{i \theta}\right), 0<\theta<2 \pi$, will do), it follows from the boundary behavior of $z g_{a}^{\prime}(z) / g_{a}(z)$ that $g_{a}$ maps $K$ onto the complex plane minus a set

$$
\{z:|z| \geqq d, \pi-\psi \leqq \arg z \leqq \pi+\psi\} \quad\left(0<\psi<\pi, \frac{1}{4}<d<1\right) .
$$

A straightforward but long computation yields the identity

$$
\begin{aligned}
\log \frac{g_{a}(z)}{z}= & \int_{0}^{z}[F(w)-1] w^{-1} d w \\
= & 2 b \log \left\{\left[\left(\frac{1+2 a z+z^{2}}{(1-z)^{2}}\right)^{1 / 2}+b \frac{1+z}{1-z}\right](1+b)^{-1}\right\} \\
& +2 \log 2\left[\left(1+2 a z+z^{2}\right)^{1 / 2}+1+z\right]^{-1}
\end{aligned}
$$


where $a=2 b^{2}-1$. From (3.1) and (3.2) we find that

$$
d=|g(-1)|=\left[(1+b)^{1+b}(1-b)^{1-b}\right]^{-1}
$$

and

$$
\psi=\pi(1-b)
$$

Let $r_{1}=r_{1}(a)$ be the first positive root of the equation

$$
1-r g_{a}^{\prime \prime}(-r) / g_{a}^{\prime}(-r)=0 \text {. }
$$

Then $r_{1} \geqq r_{0}$ and hence $d^{*} \leqq d_{1}=\left|g_{a}\left(-r_{1}\right)\right|$. We note that $\lim _{a \rightarrow 1} d_{1} / d=\frac{2}{3}$ and $\lim _{a \rightarrow 1}(d / d a)(d)=\infty$. As in Example 1, these facts can be used to show that the two-thirds conjecture is false. Here, however, we are interested only in an explicit value of $d_{1} / d$. To obtain this we first find that

$$
1+\frac{z g_{a}^{\prime \prime}(z)}{g_{a}^{\prime}(z)}=\frac{\left(1+2 a z+z^{2}\right)^{3 / 2}+z(1+a)(1+z)}{(1-z)\left(1+2 a z+z^{2}\right)}
$$

Hence $r_{1}$ is the first positive root of $\left(1-2 a r+r^{2}\right)^{3 / 2}-r(1+a)(1-r)=0$ or equivalently of the sixth degree equation

$$
\begin{aligned}
1-6 a r+\left(2-2 a+11 a^{2}\right) r^{2} & +2(1-4 a)\left(1+a^{2}\right) r^{3} \\
+ & \left(2-2 a+11 a^{2}\right) r^{4}-6 a r^{5}+r^{6}=0 .
\end{aligned}
$$

Using the substitution $u=r+r^{-1}$ we obtain a cubic equation in $u$. Solving this cubic equation we get

$$
r_{1}(a)=\left[u_{1}-\left(u_{1}^{2}-4\right)^{1 / 2}\right] / 2,
$$

where

with $A=(1+a)^{2}$.

$$
\begin{aligned}
u_{1} & =x+2 a, \quad x=2(A / 3)^{1 / 2} \cos (\theta / 3) \\
\theta & =\cos ^{-1}[(-3 \sqrt{ } 3)(1-a) /(1+a)]
\end{aligned}
$$

Using (3.2), (3.3), (3.5) and (3.6) we can calculate $d_{1} / d$ for a given value of $a, 0.68 \leqq a \leqq 1$. A close approximation to the minimum of $d_{1} / d$ is $0.644 \cdots$ given by $a=0.89$. From (3.4) it follows that $\psi \approx 0.03 \pi$ for this function.

The authors wish to thank Mr. David Blankenship for the use of his H.P. 35 Calculator to obtain the approximations given in this paper.

\section{REFERENCES}

1. E. Gray and A. Schild, A new proof of a conjecture of Schild, Proc. Amer. Math. Soc. 16 (1965), 76-77. MR 30 \#2136.

2. W. K. Hayman, Multivalent functions, Cambridge Tracts in Math. and Math. Phys. no. 48, Cambridge Univ. Press, Cambridge, 1958. MR 21 \#7302. 
3. Z. Lewandowski, Nouvelles remarques sur les théorèmes de Schild relatifs d̀ une classe de fonctions univalentes (Démonstration d'une hypothèse de Schild), Ann. Univ. Mariae Curie-Skłodowska Sect. A 10 (1956), 81-94. MR 20 \#1778.

4. C. McCarty and D. Tepper, A note on the $2 / 3$ conjecture for starlike functions, Proc. Amer. Math. Soc. 34 (1972), 417-421.

5. A. Schild, On a problem in conformal mapping of Schlicht functions, Proc. Amer. Math. Soc. 4 (1953), 43-51. MR 14, 861.

6. T. Suffridge, $A$ coefficient problem for a class of univalent functions, Michigan Math. J. 16 (1969), 33-42. MR 39 \#1646.

Department of Mathematics, University of Kentucky, Lexington, Kentucky 40506 (Current address of J. L. Lewis)

Current address (Roger W. Barnard): Department of Mathematics, Texas Tech. University, Lubbock, Texas 79409 